\title{
TEORIZAÇÃO EM EDUCAÇÃO SEXUAL A PARTIR DA GROUNDED THEORY
}

\author{
TEORIZACIÓN EN EDUCACIÓN SEXUAL A PARTIR DE GROUNDED THEORY
}

THEORIZATION IN SEXUAL EDUCATION FROM THE GROUNDED THEORY

\author{
Claudionor Renato da Silva ${ }^{1}$
}

\begin{abstract}
RESUMO: Teorização em educação sexual trata-se de produção de conhecimento que, a partir de pesquisas indutivas, como as que se utilizam da metodologia Grounded Theory (GT), originam interpretações da realidade, em temáticas específicas, como, por exemplo, gênero, sexualidade infantil, sexualidade do adolescente, formação de professores, currículo, gravidez na adolescência, etc. O objetivo deste artigo é abordar a metodologia GT para produção de teorização(ões) na área da educação sexual, utilizando-se para isso de um exemplo de pesquisa de doutoramento em sexualidade infantil que apresenta o formato e as características de uma teorização com esta metodologia, objetivando, acima de tudo, a formação de pesquisadores iniciantes na área da educação sexual, na graduação e na pós-graduação.
\end{abstract}

PALAVRAS-CHAVE: Grounded theory. Teorização. Educação sexual.

RESUMEN: La teorización en educación sexual se trata de producción de conocimiento que, a partir de investigaciones inductivas, como las que se utilizan de la metodología Grounded Theory $(G T)$, ellos originan interpretaciones de la realidad, en temáticas específicas, como por ejemplo género, sexualidad infantil, sexualidad del adolescente, formación de profesores, currículo, embarazo en la adolescencia, etc. El objetivo de este artículo es abordar la metodología GT para la producción de teorización en el área de la educación sexual, utilizando para ello un ejemplo de investigación de doctorado en sexualidad infantil que presenta el formato y las características de una teorización con esta metodología, objetivando, por encima de todo, la formación de investigadores iniciantes en el área de la educación sexual, en la graduación y en el postgrado.

PALABRAS CLAVE: Grounded theory. Teorización. Educación sexual.

ABSTRACT: Theorization in sexual education is a production of knowledge that, based on inductive researches, such as those using the Grounded Theory (GT) methodology, originate interpretations of reality in specific themes such as gender, child sexuality, adolescent sexuality, teacher training, curriculum, adolescent pregnancy, etc. The purpose of this article is to approach the GT methodology for the production of theorization(s) in the area of sexual education, using an example of a doctoral research in child sexuality that presents the format and characteristics of a theorization with this methodology, aiming, above all, the training of new researchers in the area of sexual education, in undergraduate and postgraduate studies.

${ }^{1}$ Universidade Federal de Goiás, (UFG), Jataí - GO - Brasil. Docente e Pesquisador do Curso de Pedagogia (Unidade de Educação) e Programa de Pós-Graduação em Educação (PPGE - Unidade de Ciências Humanas). ORCID: <http://orcid.org/0000-0003-1693-4804>. E-mail: claudionorsil@gmail.com 
KEYWORDS: Grounded theory. Theorization. Sexual education.

\section{Introdução}

Teorização na metodologia Grounded Theory (GT), de abordagem construtivista, em Charmaz (2009) está ligada à interpretação que se baseia em "[...] uma compreensão imaginativa do fenômeno estudado. [...] pressupõe: realidades múltiplas e emergentes; indeterminação; fatos e valores quando associados; a verdade como algo provisório; e a vida social como processo [...]" (CHARMAZ, 2009, p. 173).

Teorizar na GT é produzir a construção de compreensões abstratas dos fenômenos sociais estudados, acessados e pesquisados, algo muito longe de construções teóricas ou modelos teóricos acabados, definitivos, absolutos.

A teorização na GT versus produção de teoria tem sido o debate, tanto nos Estados Unidos, onde nasceu a metodologia, quanto na Europa e Austrália, atualmente, entre os pesquisadores da GT (MORSE et al., 2016; WALSCH et al., 2015; BIRKS; MILLS, 2015; TAROZZI, 2011; CLARKE, 2003; FERNANDES; GONÇALVES, 2001).

No interior da metodologia GT, teorização assume a acepção apresentada por Machado (2003), de investigação, especulação; a concepção de teorização na GT advém dos estudos de Robert Merton com a Teoria de Médio Alcance (TMA), detalhada na Introdução ao livro Theory and Social Structure, publicado pela primeira vez em 1949.

A teorização, na abordagem construtivista da GT, é um sinônimo de TMA, pois, para Merton, a construção de uma teoria se faz por níveis de abstração e, por passar pela subjetividade do pesquisador, do contexto de que se retira o dado ou a observação do fenômeno, ela, a observação a ser teorizada, sempre será de "médio" alcance e jamais de alcance "total"; é momentânea, transitória, é interpretativa.

Para as teorizações em GT utilizam-se as ferramentas das relações, comparações e inflexões para construir conceitos. Esse recurso é recorrente em todo o processo da metodologia do GT. A teorização é sempre uma aproximação inicial da realidade empírica e caracteristicamente interpretativa, e esta é a opção escolhida por Kathy Charmaz, organizadora da abordagem construtivista no GT. Para a autora, teorização é sinônimo de interpretação, interpretação de uma realidade; a pesquisa sob o GT construtivista não está disposta à construção de um esquema teórico como produto e, portanto, uma investigação de caráter final, acabado; teoria, propriamente dita. Para Charmaz (2009) a teorização é interpretativa, sempre. 
Logo, se contrapõe, ainda que com nuanças, diretamente à definição de "teoria", elaborada, sobretudo, na área das ciências sociais (GUERRA, 2012).

Segundo Tarozzi (2011) e Charmaz (2009), é Adele Clarke (CLARKE, 2003; 2005) quem amplia a noção de teorização interpretativa no GT com a abordagem situacional do GT, o Situational Analysis.

A abordagem construtivista da GT organizada por Kathy Charmaz, aluna de Barney Glaser, criador da metodologia ao lado de Anselm Strauss, tem como principal característica (SILVA, 2017; TAROZZI, 2011) a construção de uma pesquisa conjunta e articulada, entre pesquisador e participantes, em que não somente os fatos ou fenômenos sociais são postos para análise, mas os seus significados. Por isso, a abordagem é interpretativa, teorizada, e não "teoria", simplesmente. Esse é o motivo pelo qual também Silva (2017) vem afirmar que a tradução em português por "teoria" fundamentada é equivocada e, até certo ponto, errada, pois não se prevê produção de teoria, mas a interpretação dos fenômenos sociais investigados.

Os pressupostos da teorização (TAROZZI, 2011): 1) pesquisador(a) e participantes, constroem juntos a teorização; 2) dados são produzidos e não coletados, daí a ênfase na etapa de "codificação"; 3) ênfase também nos significados e não nos fatos ou dados, em si; 4) os significados geram categorias, conceitos, sempre flexíveis e sempre ligados aos fenômenos sociais reais; 5) categorias são elevadas a conceitos através de relações entre categorias e são melhor esclarecidas no relatório de teorização.

A abordagem construtivista de Charmaz (2009) é a apresentada na Figura 1: 
Figura 1 - Metodologia construtivista da GT (CHARMAZ, 2009)

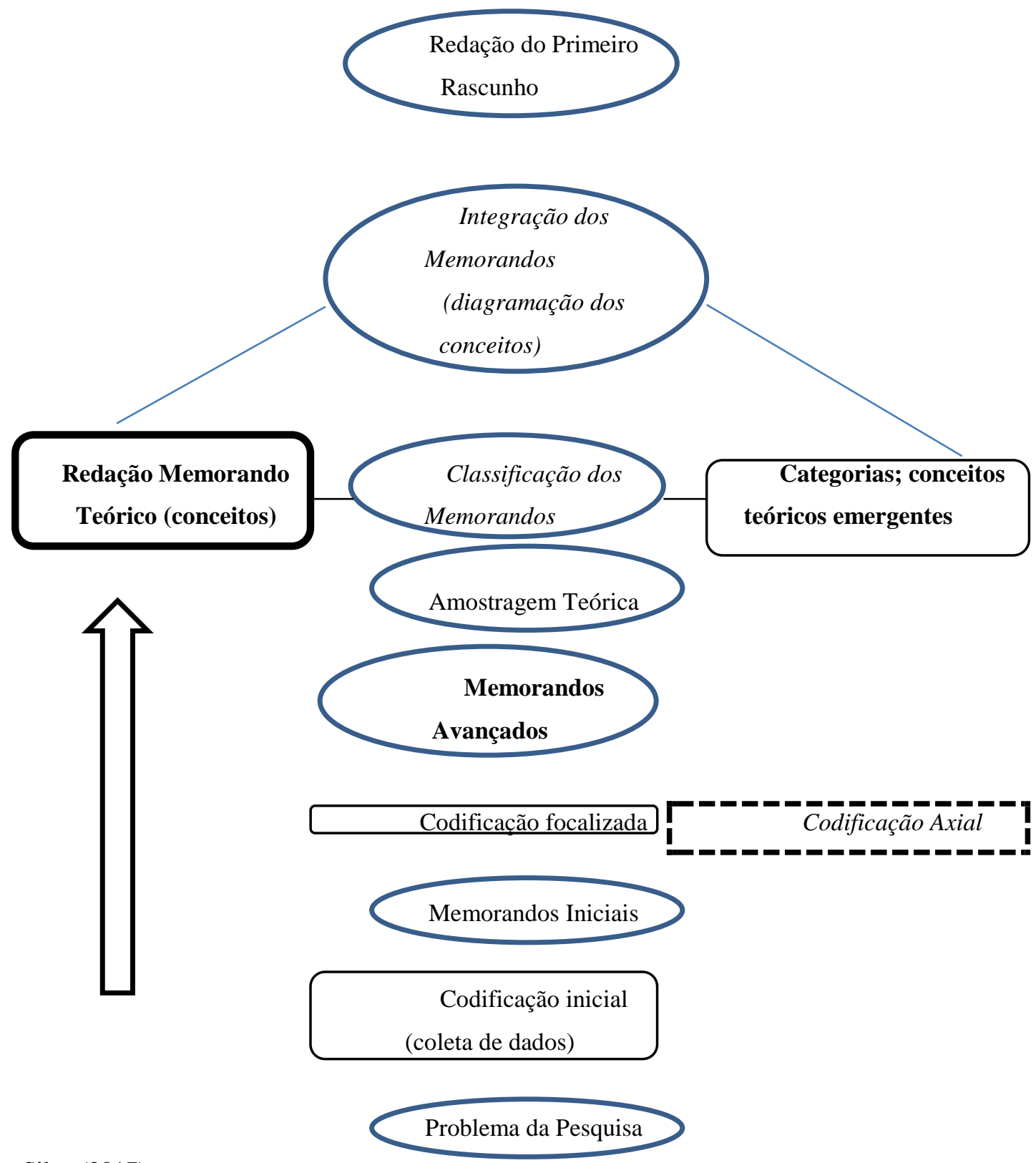

Fonte: Silva (2017)

A primeira etapa da metodologia (CHARMAZ, 2009): o problema de pesquisa. O desafio do pesquisador(a) é se perguntar, a partir do tema escolhido, por exemplo, a formação de professores em educação sexual, “o que há sobre isto?”; “O que se tem dito sobre isto?”; “O que tem a dizer os professores sobre sua formação em educação sexual?"; "O que a literatura sobre o tema apresenta?”. É importante a máxima seguinte: a pergunta de pesquisa no GT é 
bem geral, é ampla. Numa pesquisa indutiva como a GT não há uma pergunta de pesquisa restrita, ela é inicial e, fundamentalmente, ampla. E é nesta abrangência que o método se apoia na coleta de dados, chamados de "emergentes", pois irão originar/formatar as teorizações que nascem da realidade social.

Esta assertiva, segundo Silva (2017), Tarozzi (2011), Charmaz (2009), conserva a estrutura da metodologia em sua origem com Barney Glaser e Anselm Strauss, em 1967: “what's going on here?”. Por que a pergunta é ampla? Pois "[...] não pode ser definido claramente com antecedência, pois o risco seria de forçar excessivamente os dados" (TAROZZI, 2011, p. 65).

Por isso a pergunta numa pesquisa sob a GT não pode ser vista sob o prisma dedutivo de pesquisa, mas indutiva, por sua natureza metodológica.

Depois de definido o problema, que é acompanhado pelo tema ou temática que se quer pesquisar, passa-se a definir qual tipo de dados irá trabalhar e como esses dados serão coletados.

Para auxiliar os pesquisadores nessa etapa de definição do tipo de dados e a forma como serão coletados, Charmaz (2009) propõe algumas questões para se pensar: como estão distribuídos os meus dados ou como posso conseguir os dados de que preciso? Onde estão os possíveis participantes da e para a minha pesquisa de teorização? Como irei acessar meus dados, meus participantes? Essas questões são decisivas numa pesquisa sob a abordagem construtivista do GT. A falta desta definição pode comprometer os dados emergentes, logo, a teorização a que se propõe elaborar.

Uma fez definidos o tipo e o local onde os dados serão coletados é preciso definir procedimentos de alocação destes dados, o seu registro, em que, a todo tempo, até ao final das etapas da metodologia, deverão estar acessíveis ao pesquisador. Neste momento os dados são codificados, dando início ao processo de teorização.

Codificar significa, na metodologia GT, a impressão interpretativa da realidade emergente nos dados a que o pesquisador(a) tem acesso. Aqui está uma das especificidades da GT em contraposição a pesquisas de cunho qualitativo, em que categorias são construídas antes mesmo dos dados serem coletados (CHARMAZ, 2009). Codificação Inicial dos Dados, portanto, é o próprio curso interpretativo da pesquisa sob a GT: enquanto se colhem os dados, se analisam esses dados. Codificar é o mesmo que categorizar, é também, enumerar cada dado coletado e organizar, a partir de excertos, sentenças gerais, com no máximo cinco a oito palavras, que representem este "código", que, por sua vez, representa um excerto ou fragmento de texto originário do(s) dados coletados (SILVA, 2017) 
Esta codificação deve ser bem organizada e deve abranger todo o conteúdo dos dados. Lembrando que os dados são os fenômenos sociais "captados" por tipo de pesquisa, seja a entrevista, grupo focal, documentos, etnografia, enfim. Os dados devem contemplar praticamente - se possível - tudo o que existe sobre o tema. A sequência do método garante essa premissa, pela sua natureza indutiva.

Os Memorandos Iniciais são construídos de posse da codificação inicial dos dados, em que já se pode agrupar codificações e escrever sobre isso, ou seja, se elaboram os primeiros memorandos. Memorandos são, inicialmente, pequenos textos, que vão se estruturando ao longo do processo de codificação dos dados e sequência das etapas da metodologia GT. Eles podem ser aumentados, aprofundados ao longo da pesquisa, ou podem ser organizados e arquivados, conservando e mantendo suas estruturas na forma de versões sequenciais. Nesse processo, os conceitos e as categorias emergem e poderão já ir aparecendo e sendo organizados como teorização, para aprofundamentos antes da organização de memorandos avançados e do próprio Relatório da Teorização. Esses memorandos iniciais vão sendo incorporados pela atividade criativa do pesquisador(a) até serem escritos os memorandos avançados.

A Codificação Focalizada aprofunda as codificações iniciais. Charmaz (2009) também chama esta codificação de "codificação saturada". Nesta codificação o pesquisador(a) se pergunta: "O que há ainda sobre este assunto?” ou "Que aprofundamentos sobre isto ainda existe?”. Há o ensejo, caso necessário, do retorno do pesquisador(a) aos participantes da pesquisa, onde os dados foram coletados, para se questionar e validar seus "achados" iniciais, reforçar ideias dos seus memorandos, enfim, co-construir a teorização junto aos seus participantes da pesquisa.

A Codificação Axial se caracteriza por uma estruturação de relações entre categorias e subcategorias, uma espécie de mapa conceitual complexo. Elege-se uma categoria central que se classifica como eixo central e, em torno deste eixo se organizam categorias "anexas" num formato circular que represente forte interrelação das categorias, dos conceitos e da teorização em curso propriamente dita. A codificação axial representa a demarcação de categorias macro, interligando e evidenciando categorias micro, todas relacionadas entre si.

Nos Memorandos Avançados aqueles memorandos iniciais se expandem. As categorias agrupadas já compõem conceitos sensibilizadores, imaginativos e construídos pela e na abstração do pesquisador(a).

A Amostragem teórica é o momento em que o Relatório estará sendo construído em sua estrutura mais simples e inicial, e, agora sim, é permitido olhar o que se tem produzido sobre o tema; é hora de "testar" a originalidade, diga-se assim, da produção do pesquisador(a) do GT. 
É hora de esclarecer e explicitar a "coerência” das abstrações teorizadas até o momento. Ajuda também a explicar melhor os conceitos, a teorização em construção. A amostragem teórica, também chamada por Charmaz (2009) de "codificação teórica", alimenta a teorização de forma a complementar o Relatório e refinar "intelectualmente" a teorização.

"Classificar Memorandos" é a tarefa da metodologia em que se faz a organização lógica dos memorandos construídos na pesquisa e envolve três ações: 1) dar nome ao memorando e descrever seu conteúdo e abrangência; 2) representá-lo graficamente e 3) integrar os memorandos elaborados na pesquisa. Esta etapa acontece ao mesmo tempo em que o memorando está sendo construído/escrito.

A Integração dos Memorandos envolve a organização prévia do Relatório, a teorização propriamente dita. A Redação do Primeiro Rascunho é a etapa do Relatório em si, em que a teorização é finalmente apresentada. Aqui são exigidas qualidade de escrita, sustentação dos dados que originaram a teorização e, sobretudo as "testagens" da validade da mesma, com os crivos da credibilidade, originalidade, ressonância e utilidade, que serão comentadas na seção seguinte a partir do exemplo de uma pesquisa sobre sexualidade infantil em que Silva (2015) aplica a metodologia GT para gerar teorização.

\section{Um exemplo de teorização em educação sexual com a GT: um estudo sobre sexualidade infantil}

Um exemplo de teorização em educação sexual com a metodologia GT é a pesquisa de doutoramento de Silva (2015). A estrutura seguida na pesquisa é representada na Figura 2: 
Figura 2 - O método GT seguido por Silva (2015)

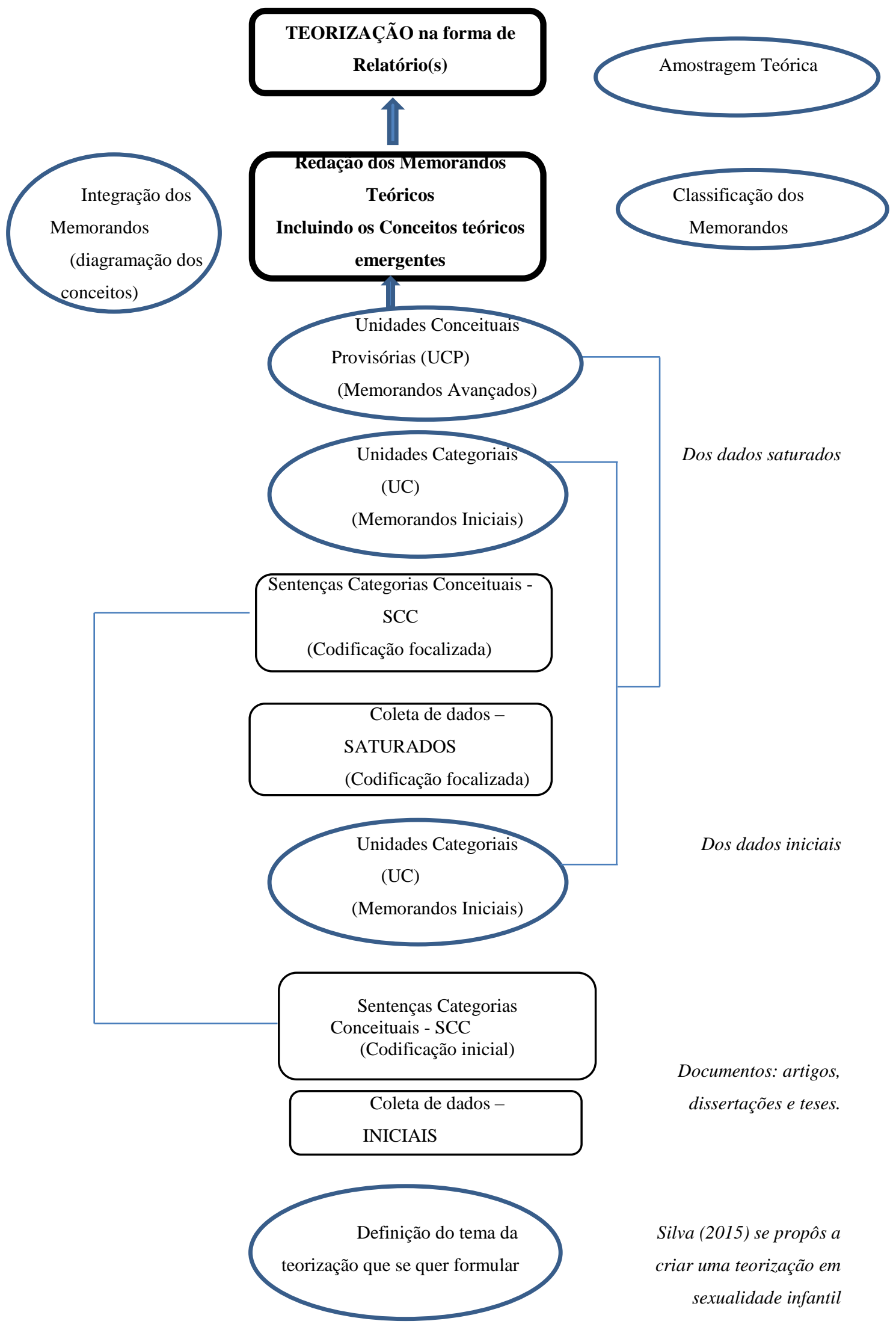

Fonte: Silva (2017). 
A modificação proposta por Silva (2017), a partir de Charmaz (2009), é dar um teor mais teórico ou de "ensaio sociológico" desde as Sentenças Categoriais Conceituais (SCC), que são, na verdade, Memorandos construídos ao longo da coleta de dados. O que isso possibilita? Possibilita maiores insights dos pesquisadores da metodologia GT, iniciantes e mais experientes, de forma que, desde o início da pesquisa e durante as etapas conseguintes, o formato de teorização vai se ajustando, se configurando, e, desta maneira, encaminhando o Relatório de Teorização.

Assim é que as Unidades Categoriais (UC) resultantes das Codificações retiradas dos dados, tanto a inicial quanto a codificada, já demonstram as linhas gerais de teorização que serão apresentadas com profundidade no Relatório de Teorização.

Na construção das Unidades Conceituais Provisórias (UCP) já está definida a teorização colocada em curso. Na pesquisa de Silva (2015), a teorização em sexualidade infantil se organizou em três relatórios: Relatório 1, Relatório 2 e Relatório 3.

Enunciado geral do Relatório de Teorização 1:

A sexualidade infantil é uma perspectiva de estudo com foco na teoria psicanalítica sobre sexualidade em Freud, mas que traz em seu bojo outras teorias da psicanálise e autores pós-freudianos até a contemporaneidade (SILVA, 2015, p. 146).

O Relatório de Teorização 2 traz o seguinte enunciado geral:

A sexualidade infantil na educação escolar deve superar o silenciamento e a resistência dos atores adultos; deve superar ações locais de pesquisa e extensão e avançar para efetividades no PPP, como política esclarecida aos pais e responsáveis e a toda comunidade escolar (SILVA, 2015, p. 194).

Por último, o Relatório de Teorização 3.

Sexualidade infantil e temática político-sociais. Políticas e gestão da sexualidade visando a proteção da criança pequena (SILVA, 2015, p. 217).

Qual a característica geral dos relatórios? A inserção da literatura em que a teorização organizada desde as Sentenças Categoriais Conceituais (SCC) foram tomando um corpus teórico até os Memorandos mais avançados que constituíram as Unidades Conceituais Provisórias (UCP). Silva (2015) afirma:

O produto final - a teorização - é o resultado de um processo que se iniciou na indexação e leitura dos dados documentais. No próprio esforço da escrita da teorização, estes dados são vitais, de modo que, para ele (para os dados) a teorização precisa retornar sempre. O caminho percorrido das Sentenças Categoriais às Unidades Categoriais, bem como, aos conceitos [...], 
permitiram, seguindo o método GT, a teorização aqui apresentada [...] (SILVA, 2015, p. 143).

A teorização, de modo geral formatada no Relatório, permite maior elucidação ou maior e melhor desenvolvimento dos conceitos que são chave e que se desenvolveram ao longo da aplicação da metodologia GT. Por exemplo, na pesquisa em sexualidade infantil, a teorização carrega, como característica, no Relatório 1, um maior desenvolvimento do conceito de “desenvolvimento biopsicossexossocial”, em que se dá um maior destaque ao desenvolvimento sexual infantil, mesmo que esta dimensão esteja explícita no interior dos estudos biológicos, psicológicos e sociais. Trata-se de evidenciar, para ficar claro que a sexualidade infantil e, portanto, as manifestações da sexualidade, na escola e em casa, ou em outros espaços sociais das crianças pequenas, são manifestações de seu desenvolvimento e, portanto, nenhuma anormalidade ou "padrões" a serem sufocados ou tratados com medicamentos.

$\mathrm{Na}$ conceituação do desenvolvimento biopsicossexossocial, a relação psicanálise e educação são fundamentais, permitindo que a presente tese sustente, já de antemão, que a psicanálise é o eixo central ao qual convergem o desenvolvimento humano, a educação escolar e as relações sócio-políticas (SILVA, 2015, p. 151).

O diálogo dos dados emergentes, das sentenças categoriais conceituais, da construção das unidades categoriais e conceituais, portanto, todo o conjunto das etapas da metodologia GT, ao dialogarem com a literatura, permitem uma teorização muito mais rica, em que os insigths teóricos do pesquisador(a) que utiliza a GT aliados à produção da área acabam, naturalmente, a validar a teorização.

No exemplo do Relatório 1, os dados indicaram, na quase totalidade, a base freudiana em psicanálise em se tratando da manifestação da sexualidade na educação infantil; indicaram, ainda mais, a contemporaneidade do tema, com autores pós-freudianos e outras perspectivas modificadas das produzidas por Freud, por exemplo, Melanie Klein, W. R. Bion, Anna Freud, Sandor Ferenczi, Erich Fromm, dentre outros(as).

Ao aprofundar as indicações dos dados, na etapa da redação do relatório de teorização, percebe-se que a produção fica ainda mais clarificada, estruturada, "refinada", como diz Charmaz (2009).

Uma consideração importante sobre teorizações a partir da GT que se encaminham para a área da educação sexual e que estão na pesquisa de Silva (2015) é que a teorização passa, finalmente, na etapa do relatório, pelo crivo da credibilidade, originalidade, ressonância e utilidade. Na pesquisa de Silva $(2015 ; 2017)$ a sexualidade infantil neste crivo se resume nas 
seguintes considerações pontuais, mas não totais, pois são mais amplas, apenas serão brevemente apresentadas:

- Credibilidade: os relatórios de teorização comunicam dados reais ao ambiente da escola da educação infantil; as categorias e conceitos gerados na pesquisa também comunicam a realidade do fenômeno estudado, no caso, a sexualidade na infância e nos espaços da escola.

- Originalidade: as categorias e conceitos são novos na área, por exemplo, desenvolvimento biopsicossexossocial e o conceito de didática da sexualidade, e fazem avançar o conhecimento na área da educação sexual.

- Ressonância: as categorias e conceitos podem e conseguem retratar a realidade de professores, coordenadores e gestores escolares, bem como de pais, principais atores no ambiente em que o fenômeno da sexualidade infantil ocorre, no âmbito do estudo; categorias e conceitos revelam a realidade.

- Utilidade: é possível no relatório de interpretação demarcar possibilidades de utilização no cotidiano escolar e familiar; há implicações práticas e diretas ao processo educativo e ao desenvolvimento infantil.

Desta forma, a contribuição da GT para uma área em específico ou a uma questão de pesquisa originária na realidade social possibilita maior aprofundamento e compreensão interpretativa oriunda de uma produção escrita teórica que emerge de realidades concretas e, portanto, dá vida "teórica" a algo que talvez nunca foi pensado, analisado ou percebido; desta forma, são geradas novas inquietações científicas para compreensão/interpretação mais profundas, mais amplas, desta mesma realidade.

Este é o desafio atual da GT (SILVA, 2017): o de transformar o conhecimento, transformar a prática, transformar os processos sociais, projetando um mundo melhor, que, em se tratando de educação sexual, diversidade, relações de gênero, populações LGBT, gravidez na adolescência e a liberdade do corpo, enfim, revelam a urgente necessidade de investigações interpretativas destes fenômenos.

Diante destas considerações, a teorização em sexualidade infantil na pesquisa de Silva (2015, p. 233) teve o seguinte enunciado geral:

Sexualidade Infantil se estabelece como teorização em uma única via: o da teoria psicanalítica da sexualidade. As subvias centrais e necessárias à teorização são duas: a primeira, o cotidiano escolar e a segunda é o das efetividades das políticas de proteção à criança na sociedade e sua gestão. 
Atualmente, a pesquisa sobre teorizações em sexualidade infantil com contribuições à educação sexual sob a GT, realizada pelo autor, projeta regiões de fronteira à psicanálise contemporânea, por exemplo, fronteiras com a filosofia de Judith Butler e Gilles Deleuze.

\section{Considerações finais}

Teorização em educação sexual com a GT é uma aposta por maior contribuição à área, em estudos de cunhos teóricos e de ensaios que se tornem subsídios, no formato de fundamentos, para outras pesquisas, ou base de reflexões conceituais no interior da área. Tensões, rupturas, inovações, diálogos e contra diálogos, mas que sempre estejam ao lado e no objetivo último do desenvolvimento humano em sua emancipação, no sentido mais amplo possível. A GT gera teorização, interpretação da realidade a partir de dados empíricos; no tocante à educação sexual, uma importante opção metodológica.

Espera-se que o presente artigo contribua para a formação de pesquisadores iniciantes na GT, desde a graduação, em diferentes licenciaturas, até a pós-graduação stricto sensu.

Também se espera que sejam gerados projetos de pesquisa e de extensão no campo da educação sexual, objetivando teorizações que se enquadrem na perspectiva das inovações educacionais que levem em conta as práticas cotidianas escolares no formato de teorizações, interpretações da realidade em relação à sexualidade e ao gênero.

Ao lado destas "esperas", aponto para o desafio de geração de produtos educacionais na graduação e na pós-graduação profissional, no sentido de pesquisas sob o GT que atendam a geração de teorizações e inovação educacional, que são muito bem-vindas à educação básica e à formação continuada de professores, tendo como eixo principal a educação sexual emancipatória. Uma educação emancipatória que eu diria ser, acima de tudo, uma educação "paralela", uma prática pedagógica e teórica transgressora à institucionalizada, demarcada hoje pela BNCC (Base Nacional Comum Curricular), demarcada também pela "falácia" da ideologia de gênero e da escola sem partido; uma prática pedagógica e teórica de enfrentamento intelectual e político em educação sexual, contra a pior das falácias retrógradas atuais, como "menino usa azul e menina usa rosa". Urge uma prática transgressora em educação sexual contra os retrocessos a que se assistem na educação brasileira. 


\section{REFERÊNCIAS}

BIRKS, M.; MILLS, J. Grounded Theory: a practical guide. 2. ed. London: SAGE, 2015. ISBN 978-1-446-62957-86.

CHARMAZ, K. A construção da teoria fundamentada. Guia prático para análise qualitativa. Porto Alegre: Artmed, 2009. ISBN 975-85-363-1998-5.

CLARKE, A. E. Situational analysis - grounded theory mapping after the postmodern turn. Symbolic Interaction, v. 26, n. 4, p. 553-576, 2003. ISNN 1533-8665.

CLARKE, A. E. Situational analysis: grounded theory after the postmodern turn. Thousand Oaks, CA: Sage, 2005. ISBN 978-0-761-93056-3.

FERNANDES, E.; GONÇALVES, Ó. Encontro de narrativas terapêuticas: memórias do terapeuta activadas durante o processo de recordação do cliente. Revista Internacional de Psicología Clínica e de la Salud, v. 1, n. 1, p. 53-72, 2001. ISSN 15767329.

GUERRA, L. A relação entre teoria e pesquisa nas ciências sociais. Raízes, v. 32, n. 1, jan./jun., 2012. ISSN 2358-8705.

MACHADO, J. P. Dicionário Etimológico da Língua Portuguesa. Lisboa: Livros Horizonte, 2003. ISBN 978-85-86368-17-2.

MORSE, J. M. et al. Developing Grounded Theory: the second generation. London: Routledge, 2016. ISBN 978-0-203-34154-4.

SILVA, C. R. da. Proposta teórico-interpretativa em sexualidade infantil: contribuição à educação sexual a partir da Grounded Theory. 2015. 341f. Tese. Doutorado. Programa de Pós-Graduação em Educação Escolar, Universidade Estadual "Júlio de Mesquita Filho", Faculdade de Ciências e Letras de Araraquara, Câmpus de Araraquara. Araraquara, SP, 2015.

SILVA, C. R. da. Grounded Theory: a abordagem construtivista para pesquisas em educação sexual no Brasil. Uma proposta de formação de novos(as) pesquisadores(as). 1. ed. Araraquara, SP: Amazon, 2017. ISBN 978-85-924841-0-1. Disponível em: https://www.amazon.com.br/GROUNDED-THEORY-abordagem-construtivista-pesquisasebook/dp/B0752CWHTK/ref=sr_1_1?ie=UTF8\&qid=1548926937\&sr=8$1 \&$ keywords=grounded+theory+claudionor. Acesso em: 15 dez. 2019.

TAROZZI, M. O que é a Grounded Theory? Metodologia de pesquisa e de teoria fundamentada nos dados. Petrópolis, RJ: Vozes, 2011. ISBN 978-85-326-4188-5.

WALSH, I. et al. What Grounded Theory Is... A critically reflective conversation among scholars. Organizational Research Methods, v. 18, n. 4, p. 582-599, 2015. ISSN 10944281. 


\section{Como referenciar este artigo}

SILVA, Claudionor Renato da. Teorização em educação sexual a partir da Grounded Theory. Revista Ibero-Americana de Estudos em Educação, Araraquara, v. 14, n. esp. 2, p. 14271440, jul., 2019. E-ISSN: 1982-5587. DOI: 10.21723/riaee.v14iesp.2.12609

Submetido em: 30/09/2018

Revisões requeridas: 20/02/2019

Aprovado em: 30/04/2019

Publicado em: 26/06/2019 